\title{
The Use of a Modified Blood Pressure Device to Detect Atrial Fibrillation in Outpatients
}

\author{
Haroldas Razvadauskas ${ }^{1}$, , Laima Jankauskiené ${ }^{2}$, Albinas Naudžiūnas ${ }^{1}$ \\ ${ }^{1}$ Department of Internal Medicine, Lithuanian University of Health Sciences, Kaunas, Lithuania \\ ${ }^{2}$ Department of Cardiology, Kaunas Clinical Hospital, Kaunas, Lithuania
}

Email address:

haroldas.raz@gmail.com (H. Razvadauskas), ljankauskiene5@gmail.com (L. Jankauskienè), albinas.naudziunas@lsmuuni.lt (A. Naudžiunas) ${ }^{*}$ Corresponding author

\section{To cite this article:}

Haroldas Razvadauskas, Laima Jankauskienè, Albinas Naudžiūnas. The Use of a Modified Blood Pressure Device to Detect Atrial Fibrillation in Outpatients. Cardiology and Cardiovascular Research. Vol. 4, No. 2, 2020, pp. 47-51. doi: 10.11648/j.ccr.20200402.13

Received: March 6, 2020; Accepted: March 24, 2020; Published: April 21, 2020

\begin{abstract}
The prevalence and integration of self-monitoring devices is increasing across healthcare systems, [1] and this is leading to patients taking greater responsibility for their health and well-being. Our aim was to compare two groups of patients with reoccurring atrial fibrillation (AF) and their ability to detect AF with the Microlife BP A6 device. The groups were divided according to being symptomatic or asymptomatic for AF. Method: The study was conducted for 3 years. The patients were selected from Kaunas clinical hospital in Kaunas, Lithuania. In total, 60 people took part in the study. The major criterion for inclusion was based on patients having AF upon arrival. During the period of hospitalisation, they were restored to having sinusoidal rhythm (SR). There were two groups of patients in the study. The first group had clear symptoms during the onset of rhythm disturbance and the second group was asymptomatic. All patients under the age of 18 years and those who did not sign the agreement to participate were excluded from the study. The patients were contacted every 6 months by phone call and a questionnaire was filled. The statistical analysis was performed using SPSS 23 and Microsoft Office Excel 2007 software. The results were deemed statistically significant when $p<0.05$. Results: There is a statistical difference between the symptomatic and asymptomatic AF patients as compared to how useful they found the device and its effect on anxiety $(\mathrm{p}<0.05)$. There was no statistical difference between the two groups on how frequently they used the device. The research also shows that there was no difference based on gender, education levels. Conclusion: There is a statistically significant portion of AF asymptomatic patients that find the Microlife BP A6 "very useful" and an anxiety reliever as compared to patients that are symptomatic for the condition. Both groups have statistically equally poor adherence in using the device at home.
\end{abstract}

Keywords: Atrial Fibrillation, AF, Secondary Prevention of Atrial Fibrillation, Stroke, Self-monitoring Device, Microlife BP A6

\section{Introduction}

Self-monitoring devices are starting to play a larger role in modern healthcare systems - especially in cardiology [1, 2]. A growing problem in cardiology is the rising number of people suffering from atrial fibrillation (AF) and this number is expected to increase in the E. U. [3]. Patients that develop AF have a fivefold greater likelihood of suffering from brain stroke [3].

Patient self-care with self-monitoring devices can be greatly beneficial in earlier diagnosis, prevention of complications and in reducing healthcare costs [3,4]. There is also an indication that atrial fibrillation detection devices can even reduce anxiety levels in subjects [13].

The other problem with AF is that there is a certain group of patients who do not feel heart rhythm disturbance and the silent or asymptomatic AF prevalence is hard to assess. Though in about one - third of patients with this arrhythmia, patients are not aware of the presence of AF [10] and this can lead to a delay in detection and diagnosis. Therefore AF detection devices might play an important role in such patients.

At the same time, self-monitoring devices can present their own challenges. It is known that self-monitoring for hypertension or medication use can be lacking in patients [5]. 
Our study focused on the use of the Microlife BP A6 blood pressure monitoring device with atrial fibrillation detection. Microlife BP A6 technology has shown to effectively detect atrial fibrillation [6]. The specificity of $86 \%$ and sensitivity of 95\%.

Another study showed that the Microlife BP A6 showed a sensitivity of $95 \%$, specificity of $92 \%$ and an accuracy of $94 \%$ [8]. The effective detection of rhythm disturbances can prevent medical complications and debilitating complications such as strokes $[8,9]$.

Though the device has shown to be effective in detection, there [8] has been little research done in the ability of Lithuanian patients to use the device independently at home.

Whilst the effectiveness of self-monitoring depends on two key factors: on the device and on the patient using it, we were interested in the ability of the patients to adhere to using the device for a period of months at home. We were also interested in investigating if the device can reduce anxiety in $\mathrm{AF}$ patients.

Hence, the research was set up to investigate patients' adherence to the use of the Microlife BP A6 device over a period of a couple of years and to examine its effect on anxiety.

\section{Method}

The study was conducted for three years. The patients were selected from Kaunas clinical hospital in Kaunas, Lithuania. In total 60 people took part in the study. The major criterion for inclusion was based on patients having AF upon arrival. During the period of hospitalisation, they were restored to having sinusoidal rhythm (SR). All patients under the age of 18 years and those who did not sign the agreement to participate were excluded from the study.

There were two groups of patients in the study: one that had clear symptoms during the onset of rhythm disturbance and the second which was asymptomatic.

The research was conducted for 3 years. The patients were contacted every 6 months by phone call and a questionnaire was filled. The statistical analysis was performed on a total of
34 patients.

The statistical analysis was performed using SPSS 23 and Microsoft Office Excel 2007 software.

The results were deemed statistically significant when $\mathrm{p}<0.05$.

\section{Results}

The study included 34 patients: 18 (52.9\%) males and 16 (47.1\%) females. The age of the subjects' ranged from 53 to 91 years with a mean age of $73.09 \pm 8,781$.

There were $6(17,6 \%), 18(52,9 \%)$ and $10(29,4 \%)$ patients with primary, secondary and tertiary education, respectively. From the 34 patients, $23(67.6 \%)$ subjects felt AF and the other $11(32.4 \%)$ were asymptomatic for it.

The study was run for three years. There were 26 patients that dropped out such that there were 34 patients by the end of the study.

The statistical analysis was performed using SPSS 23 and Microsoft Office Excel 2007 software. Due to the sample size, the Shapiro- Wilk test was applied to evaluate the normality of the data distribution. Where data was not naturally distributed a Chi-square test was applied. The association between age and frequency of AF blood pressure apparatus use was evaluated using the Spearman's correlation test. The results were deemed statistically significant when $\mathrm{p}<0.05$.

Our analysis focused on symptomatic and asymptomatic AF groups. We were interested to find out how frequently the device was used and how useful the patients found the device (tables 1 and 2).

Then we looked at the possible influence of gender, the frequency of use and patients' perspective on how useful the device was (table 3 and table 4 ).

We compared the influence of age on the frequency of use and patients' perspective on how useful the device was (table 5). The possible influence of education levels on use frequency and patients' perspective on how useful the device was also analysed (table 6 and table 7 ).

Finally, we examined if patients self-reported reduced anxiety levels (table 8).

Table 1. The frequency of use of the BP device for symptomatic and asymptomatic AF subjects.

\begin{tabular}{|c|c|c|c|c|c|}
\hline \multirow{2}{*}{ Groups } & \multicolumn{4}{|c|}{ How frequently do you use the BP measuring device per week? } & \multirow{2}{*}{$\begin{array}{l}\chi^{2} \text { and } p \text { sig. } \\
\text { values }\end{array}$} \\
\hline & Do not use & Use less than 7 per week & Use 7-14 per week & Use 14 per week & \\
\hline Symptomatic for $\mathrm{AF} \mathrm{n}=23$ & $2(8.7 \%)$ & $12(52.2 \%)$ & $1(4.3 \%)$ & $8(34.8 \%)$ & $\chi^{2}=0.358$ \\
\hline Asymptomatic for $\mathrm{AF} \mathrm{n}=11$ & $1(9.1 \%)$ & $5(45.5 \%)$ & $1(9.1 \%)$ & $4(36.4 \%)$ & $\mathrm{p}=0.949$ \\
\hline
\end{tabular}

There is no statistical difference in how frequently the devices are used between asymptomatic and symptomatic AF patients $(\mathrm{p}>0.05)$.

Table 2. The subjects' perception of how useful the BP device as compared to symptomatic and asymptomatic AF patients groups.

\begin{tabular}{|c|c|c|c|c|c|}
\hline \multirow{2}{*}{ AF groups } & \multicolumn{4}{|c|}{ Was the BP device useful? } & \multirow{2}{*}{$\begin{array}{l}\chi^{2} \text { and } p \text { sig. } \\
\text { values }\end{array}$} \\
\hline & No & Not a lot & Average usefulness & Very useful & \\
\hline Symptomatic $\mathrm{AF} n=23$ & $6(26.1 \%)$ & $4(17.4 \%)$ & $6(26.1 \%)$ & $7(30.4 \%)$ & $\chi^{2}=8.437$ \\
\hline Asymptomatic AF $n=11$ & $1(9.1 \%)$ & $1(9.1 \%)$ & $0(0.0 \%)$ & $9(81.8 \%)$ & $\mathrm{p}=0.038$ \\
\hline
\end{tabular}

There is a statistical difference between symptomatic and asymptomatic AF patients and their perception on how useful they found the device to be $(\mathrm{p}<0,05)$. The patients that were asymptomatic for AF found the BP device more useful in contrast to symptomatic AF subjects. 
Table 3. The frequency of the BP device use as compared to gender.

\begin{tabular}{llllll}
\hline \multirow{2}{*}{ Gender } & \multicolumn{3}{l}{ How frequently do you use the BP measuring device per week? } & \multicolumn{2}{l}{$\chi^{2}$ and $\mathrm{p}$ sig. } \\
\cline { 2 - 5 } & Do not use & Less than 7 per week & Between 7 to 14 per week & 14 times per week \\
\hline Male $\mathrm{n}=18$ & $1(5.6 \%)$ & $11(61.7 \%)$ & $0(0.0 \%)$ & $6(33.3 \%)$ & $\chi^{2}=3.699$ \\
Female $\mathrm{n}=16$ & $2(12.5 \%)$ & $6(37.5 \%)$ & $2(12.5 \%)$ & $6(37.5 \%)$ & $\mathrm{p}=0.296$ \\
\hline
\end{tabular}

There is no statistically significant difference between genders and the frequency of use of the BP device ( $p>0.05)$. It seems that both men and women used the device equally frequently.

Table 4. The patient's perspective on how useful the BP device as compared to gender.

\begin{tabular}{llllll}
\hline \multirow{2}{*}{ Gender } & \multicolumn{2}{l}{ Was the BP device useful? } & & & \multicolumn{2}{l}{$\chi^{2}$ and $p$ sig. } \\
& No & Not a lot & Average usefulness & Very useful & \\
\hline Male $\mathrm{n}=18$ & $4(22.2 \%)$ & $3(16.7 \%)$ & $2(11.1 \%)$ & $9(50.0 \%)$ & $\chi^{2}=1.146$ \\
Female $\mathrm{n}=16$ & $3(18.8 \%)$ & $2(12.5 \%)$ & $4(25.0 \%)$ & $7(43.8 \%)$ & $\mathrm{p}=0.766$ \\
\hline
\end{tabular}

There is no statistically significant difference between gender and patient's perspective on the usefulness of the device $(\mathrm{p}>0.05)$.

Table 5. The relationship between age and BP device frequency of use and perspective of the patient on how useful the device is.

\begin{tabular}{lll}
\hline & & Age \\
\hline The frequency of use of the device & $\mathrm{r}$ & -0.022 \\
& $\mathrm{p}$ & 0.900 \\
The patients' perspective on how useful the BP device is & $\mathrm{r}$ & -0.217 \\
\hline
\end{tabular}

The results show that there is a negative correlation between age and frequency of use of the BP device. The older the patient the less he or she uses it. Though this finding is statistically insignificant $p>0.05$. There is also a negative correlation between the perspective of the patient on how useful the device is and the subject's age. This is statistically insignificant ( $\mathrm{p}>0.05)$.

Table 6. The frequency of use of the BP device as compared to patient education levels.

\begin{tabular}{|c|c|c|c|c|c|}
\hline \multirow{2}{*}{ Education level } & \multicolumn{4}{|c|}{ How many times do you use the device per week? } & \multirow{2}{*}{$\begin{array}{l}\chi^{2} \text { and p sig. } \\
\text { values }\end{array}$} \\
\hline & Do not use & Less than 7 per week & Between 7 to 14 per week & 14 times per week & \\
\hline Primary $n=6$ & $1(16.7 \%)$ & $4(66.7 \%)$ & $0(0.0 \%)$ & $1(16.7 \%)$ & \multirow{3}{*}{$\begin{array}{l}\chi^{2}=6.411 \\
p=0.378\end{array}$} \\
\hline Secondary $n=18$ & $2(11.1 \%)$ & $6(33.3 \%)$ & $2(11.1 \%)$ & $8(44.4 \%)$ & \\
\hline Higher education $n=10$ & $0(0.0 \%)$ & $7(70.0 \%)$ & $0(0.0 \%)$ & $3(30.0 \%)$ & \\
\hline
\end{tabular}

There is no statistically significant difference between education levels and frequency of use of the device $(p>0.05)$.

Table 7. The patients' perspective on how useful the BP device is compared to their education levels.

\begin{tabular}{|c|c|c|c|c|c|}
\hline \multirow{2}{*}{ Education } & \multicolumn{4}{|c|}{ Was the BP device useful? } & \multirow{2}{*}{$\begin{array}{l}\chi^{2} \text { and } p \text { sig } \\
\text { values }\end{array}$} \\
\hline & No & Not a lot & Average usefulness & Very useful & \\
\hline Primary $n=6$ & $2(33.3 \%)$ & $4(66.7 \%)$ & $0(0.0 \%)$ & $0(0.0 \%)$ & \multirow{3}{*}{$\begin{array}{l}\chi^{2}=19.651 \\
p=0.003\end{array}$} \\
\hline Secondary $n=18$ & $3(16.7 \%)$ & $1(5.6 \%)$ & $3(16.7 \%)$ & $11(61.1 \%)$ & \\
\hline Higher education $n=10$ & $2(20.0 \%)$ & $0(0.0 \%)$ & $3(30.0 \%)$ & $5(50.0 \%)$ & \\
\hline
\end{tabular}

There is a statistically significant difference between different education levels and on the perception of how useful the BP device is $(\mathrm{p}<0.05)$.

Table 8. The comparison of anxiety levels reported by both groups of patients.

\begin{tabular}{llll}
\hline \multirow{2}{*}{ AF groups } & Did the device reduce anxiety? & & \multicolumn{1}{c}{$\chi^{2}$ and $\mathbf{p ~ s i g . ~}$} \\
\cline { 2 - 4 } & No & Yes & $\chi^{2}=7.886$ \\
\hline Symptomatic $\mathrm{AF}(\mathrm{n}=23)$ & $16(69.6 \%)$ & $7(30.4 \%)$ & $\mathrm{p}=0.009$ \\
Asymptomatic $\mathrm{AF}(\mathrm{n}=11)$ & $2(18.2 \%)$ & $9(81.8 \%)$ & \\
\hline
\end{tabular}

There is a statistically significant difference between symptomatic and asymptomatic patients and anxiety felt $(\mathrm{p}=$ 0.009).

\section{Discussion}

There has been little research done on the diagnostic sensitivity and specificity of self-monitoring AF devices. Such devices have become more prevalent as discussed by H. R. Marston et al. in mobile self-monitoring of an ECG device to diagnose arrhythmia. Though little research has been done towards the adherence of patients in using them [12], our research sought to correct this.

The study shows that patients poorly adhere to 
self-monitoring with only $35 \%$ of patients in both groups (symptomatic and asymptomatic) self-monitoring as recommended (Table 1). This result is in line with Michael Burnier and Brent M. Egan research article; Adherence in Hypertension: A Review of Prevalence, Risk Factors, Impact, and Management [11]. The research shows suboptimal compliance in using antihypertensive drugs among subjects with primary hypertension. Our research indicates that poor adherence extends to self-monitoring too.

The patients were informed of this fact, told about complications such as stroke and were provided with an information leaflet.

When comparing the two groups of the study, the patient group that felt signs and symptoms associated with AF as compared to the asymptomatic group, we saw a clear difference in the usefulness of the Microlife A6 device. There was a statically significant portion of asymptomatic patients that found the apparatus "very useful" as compared to patients that were symptomatic for the condition. The percentage was $\sim 82 \%$ and $\sim 30 \%$ respectively and the $p$-value was 0.038 (Table 2). There was no statistical difference between how frequently both genders used the device and how useful they found the device to be $p>0.05$ (Table 3 and Table 4).

What is peculiar about both groups of patients as mentioned before is that they used the device equally infrequently. This is contrary to logic that if one group would find the device more useful, they would use it more frequently. A larger study needs to be carried out to better analyse this result.

Looking back as to why asymptomatic patients might have found the device subjectively more useful, it could have been due to anxiety levels that are associated with not knowing about the possible new onset of rhythm disturbance. When two groups were compared on how they perceived the influence of the device on anxiety levels, $81.8 \%$ of asymptomatic for AF felt that having and using the device reduced anxiety. Whilst only $30.4 \%$ of symptomatic patients felt that the device had helped with anxiety. These results were indeed statistically significant $\mathrm{p}=0.009$ (Table 8 ).

Though education level did not influence the frequency of use of the device, (Table 6) it played a role in patients' perception of how useful the device was. There was a statistically significant difference between education levels and on the perception of how useful the BP device is ( $p=$ 0.003). Though the sample is, of course, small, it indicates that patients with secondary and higher education more frequently deemed the device as being "very useful" as compared to patients with primary education (Table 7).

One speculation why this might be the case is a fear of the unknown. Asymptomatic patients never know when they can have reoccurring AF. This can increase anxiety in the patient. Hence the device provides the patient with an ability to check their heart rhythm and reduce anxiety. This conclusion concurs with research by N. Lowres et al. [13], where researchers suggested that using an iPhone electrocardiogram might be very important in reducing anxiety. As anxiety can be one of the triggers in reoccurring atrial fibrillation, hence some therapeutic benefits could be attained by using AF self-monitoring devices by outpatients.

Though bizarrely enough asymptomatic patients did not use the device more frequently than symptomatic patients. Hence further investigations need to be performed before we can gain more insights into the use of AF self-monitoring devices.

The results show that there is a negative correlation between age and frequency of use of the BP device (Table 5). The older the patient the less he or she uses it. Though this finding is statistically insignificant $\mathrm{p}>0.05$.

This issue needs to be further investigated in larger studies as cognitive decline occurs with older age [15]. What is less known is how this could influence patients' use of AF detection devices. Therefore further studies need to be conducted on the influence of cognitive function in self-monitoring.

There is also a negative correlation between the perspective of the patient on how useful the device is and the subjects' age. This is statistically insignificant $(\mathrm{p}>0.05)$.

In summary, we can see that patients have poor adherence to self-monitoring and more needs to be done to improve it. Nonetheless, we can see devices can have usefulness beyond their intended diagnostic function. The devices seem to reduce perceived anxiety levels in AF asymptomatic patients much more than in the other group, though we state this with care as the sample size of the study was small and the patient population was homogenous.

Hence we see that self-monitoring devices have a large part to play in patients' health today and the future, yet informing patients of complications and even giving out leaflets is not enough.

We also propose that patients that are asymptomatic for reoccurring $\mathrm{AF}$ should be advised to acquire these $\mathrm{BP}$ measuring devices with an atrial fibrillation detection function.

\section{Conclusion}

The research indicates that patients' devotion to using the Microlife BPA6 device can be as low as for any anti-hypertensive drug [5]. Hence, more research is needed to understand how patients can better conform to using such self-monitoring devices. This is despite patients being informed of the importance of early self-diagnosis and the complications which can arise from AF, such as stroke.

The device seems to reduce anxiety more in patients with asymptomatic AF as compared to symptomatic patients which concurs with previous research [13]. The reduction in anxiety could have benefits in decreasing reoccurring AF [14]. Therefore, we recommend that Cardiologists and family doctors take a more proactive approach in recommending the Micfrolife BPA6 or any other such device to asymptomatic AF patients.

Our research showed that age could have a negative correlation with the use of the apparatus. However, this was statistically insignificant, and a larger study needs to be done 
to reject or accept this conclusion.

\section{Conflict of Interest Statement}

There is no conflict of interest for this study to report. The Microlife BP A6 devices were acquired with researchers funds.

\section{Acknowledgements}

We want to thank Darius Milkevičius a Microlife representative in Lithuania for assiting us with the use and maintaince of the devices during the research period.

\section{References}

[1] Sanders JP, Loveday A, Pearson N, Edwardson C, Yates T, Biddle SJ, Esliger DW. Devices for Self-Monitoring Sedentary Time or Physical Activity: A Scoping Review. J Med Internet Res 2016; 18 (5): e90.

[2] Cappuccio Francesco P, Kerry Sally M, Forbes Lindsay, Donald Anna. Blood pressure control by home monitoring: meta-analysis of randomised trials $B M J$ 2004; 329: 145.

[3] Antonio Di Carlo, Leonardo Bellino, Domenico Consoli, Fabio Mori, Augusto Zaninelli, Marzia Baldereschi, Alessandro Cattarinussi, Maria Grazia D'Alfonso, Chiara Gradia, Bruno Sgherzi, Giovanni Pracucci, Benedetta Piccardi, Biancamaria Polizzi, Domenico Inzitari, National Research Program: Progetto FAI. La Fibrillazione Atriale in Italia, Prevalence of atrial fibrillation in the Italian elderly population and projections from 2020 to 2060 for Italy and the European Union: the FAI Project, EP Europace, Volume 21, Issue 10, October 2019, Pages 1468-1475.

[4] Paulus Kirchhof, Stefano Benussi, Dipak Kotecha, Anders Ahlsson, Dan Atar, Barbara Casadei, Manuel Castella, Hans-Christoph Diener, Hein Heidbuchel, Jeroen Hendriks, Gerhard Hindricks, Antonis S Manolis, Jonas Oldgren, Bogdan Alexandru Popescu, Ulrich Schotten, Bart Van Putte, Panagiotis Vardas, ESC Scientific Document Group, 2016 ESC Guidelines for the management of atrial fibrillation developed in collaboration with EACTS, European Heart Journal, Volume 37, Issue 38, 7 October 2016, Pages 2893-2962.

[5] Vrijens B, Antoniou S, Burnier M, de la Sierra A, Volpe M.
Current Situation of Medication Adherence in Hypertension. Front Pharmacol. 2017; 8: 100. Published 2017 Mar 1.

[6] Joseph Wiesel, Lorenzo Fitzig, Yehuda Herschman, Frank C. Messineo, Detection of Atrial Fibrillation Using a Modified Microlife Blood Pressure Monitor, American Journal of Hypertension, Volume 22, Issue 8, August 2009, Pages 848852.

[7] Prati, L.; Pecchioli, V.; Germano', G.; Fedele, F. BP A6 PC (Microlife) vs help check (picolution) for atrial fibrillation screenin in the hypertension population. A comparative study between two automatic oscilloetric devices. Journal of Hypertension: June 2015 - Volume 33 - Issue - p e152.

[8] Lip GYH, Freedman B, De Caterina R, Potpara TS. Stroke prevention in atrial fibrillation: past, present and future. Thromb Haemost 2017; 117: 1230-9.

[9] Hisashi Ogawa, Yoshimori An, Syuhei Ikeda, Yuya Aono, Kosuke Doi, Mitsuru Ishii, Moritake Iguchi, Nobutoyo Masunaga, Masahiro Esato, Hikari Tsuji, Hiromichi Wada, Koji Hasegawa, Mitsuru Abe, Gregory Y. H. Lip, Masaharu Akao. Progression From Paroxysmal to Sustained Atrial Fibrillation Is Associated With Increased Adverse Events. Stroke. 2018; 49: 2301-2308.

[10] Polychronis E. Dilaveris and Harold L. Kennedy. Silent atrial fibrillation: epidemiology, diagnosis, and clinical impact. Clin Cardiol. 2017 Jun; 40 (6): 413-418.

[11] Michel Burnier, Brent M. Egan. Adherence in Hypertension. Circulation Research. 2019; 124: 1124-1140.

[12] Marston HR, Hadley R, Banks D, Duro MDCM. Mobile Self-Monitoring ECG Devices to Diagnose Arrhythmia that Coincide with Palpitations: A Scoping Review. Healthcare (Basel). 2019; 7 (3): 96. Published 2019 Aug 16.

[13] Nicole Lowres, Georgina Mulcahy, Robyn Gallagher, Saul Ben Freedman, David Marshman, Ann Kirkness, Jessica Orchard, Lis Neubeck, Self-monitoring for atrial fibrillation recurrence in the discharge period post-cardiac surgery using an iPhone electrocardiogram, European Journal of Cardio-Thoracic Surgery, Volume 50, Issue 1, July 2016, Pages 44-51.

[14] Severino P, Mariani MV, Maraone A, et al. Triggers for Atrial Fibrillation: The Role of Anxiety. Cardiol Res Pract. 2019: 1208505. Published 2019 Feb 18. doi: 10.1155/2019/1208505.

[15] Harada CN, Natelson Love MC, Triebel KL. Normal cognitive aging. Clin Geriatr Med. 2013; 29 (4): 737-752. doi: 10.1016/j.cger.2013.07.002. 